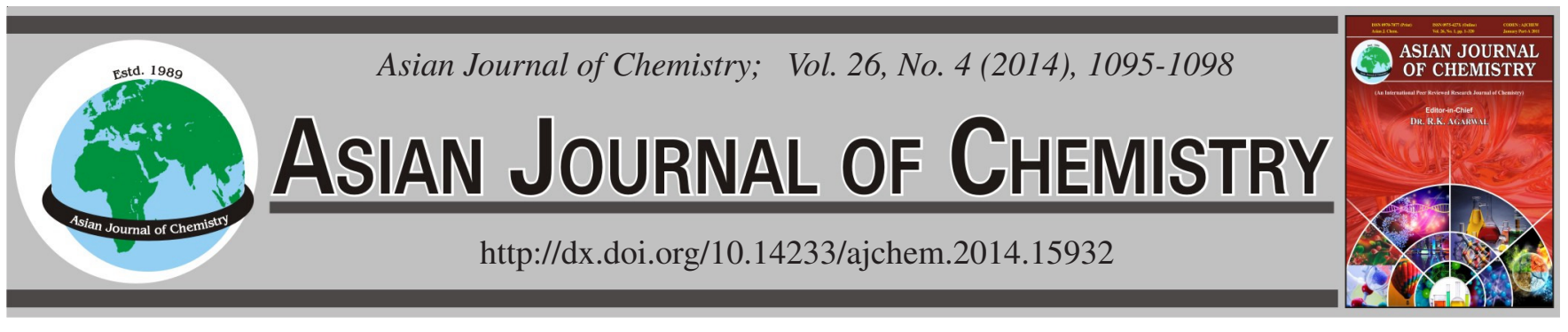

\title{
Synthesis, Spectroscopic Characterization and Thermal Studies of (E,E)-2-Hydroxy-3-methoxybenzaldehyde Azine
}

\author{
Rong Zhou ${ }^{1,2}$, Huaibo Zou ${ }^{1}$ and GuangQuan MeI ${ }^{1, *}$
}

${ }^{1}$ Key Laboratory of Jiangxi University for Applied Chemistry \& Chemical Biology, Yichun University, Jiangxi Province, P.R. China ${ }^{2}$ College of Chemistry \& Bioengineering, Yichun University, Jiangxi Province, P.R. China

*Corresponding author: Tel/Fax: +86 795 3200655; E-mail: yc_mgq@163.com

Received: 18 June 2013;

Accepted: 28 August 2013;

Published online: 15 February 2014;

AJC-14706

Hydrazone, (E,E)-2-hydroxy-3-methoxybenzaldehyde azine was synthesized and its spectroscopic characterization was studied via IR, ${ }^{1} \mathrm{H}$ NMR, MS and XRD. Its fluorescence activity and thermal stability were also analyzed.

Keywords: Hydrazone, Spectroscopic characterization, XRD, Thermal analysis.

\section{INTRODUCTION}

Hydrazones contain the azomethine group $(-\mathrm{RC}=\mathrm{N}-)$ and usually formed by the condensation of a primary amine with an active carbonyl compound ${ }^{1}$. Hydrazones are widely used as intermediates in synthesis ${ }^{2}$, and in particular in hydrazone Schiff base ligands, which are among others employed in dinuclear catalysts ${ }^{3}$. In analytical chemistry, the formation of hydrazones was extensively used as ideal reagents in detection, determination and isolation of compounds containing the carbonyl group or transition metals determinations $s^{4,5}$.

Furthermore, hydrazones demonstrate many bioactivities in the treatment of several diseases, therefore, a number of hydrazide-hydrazone derivatives have been claimed to possess interesting antibacterial ${ }^{6-7}$, antifungal ${ }^{8-9}$, anticonvulsant ${ }^{10}$, antiinflammatory $^{11}$, antimalarial ${ }^{12}$, and antituberculosis activities ${ }^{13}$. Hydrazones have also been used for different purposes such as herbicides, insecticides ${ }^{14-15}$ and plant growth regulators ${ }^{16}$. This paper contains the synthesis, characterizations and thermal stability of the hydrazone, $(E, E)$-2-hydroxy-3-methoxybenzaldehyde azine (Scheme-I).

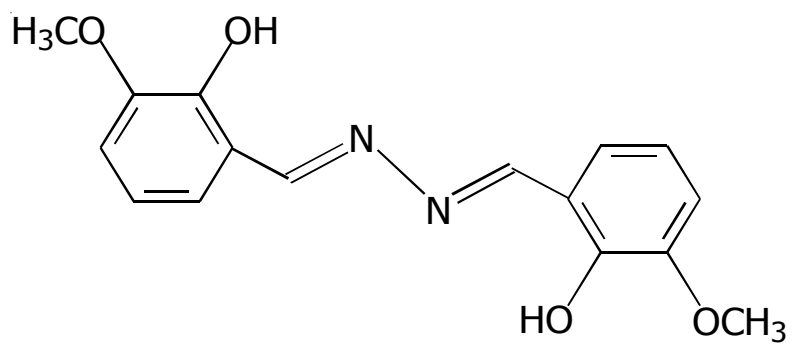

Scheme-I: $(E, E)-2$-Hydroxy-3-methoxybenzaldehyde azine

\section{EXPERIMENTAL}

2-Hydroxy-3-methoxybenzaldehyde (Fluka) and hydrazine hydrochloride (Fluka) were used as received. Analytical grade ethanol was used as solvent.

Synthesis of $(E, E)$-2-hydroxy-3-methoxybenzaldehyde azine: The $(E, E)$-2-hydroxy-3-meth-oxybenzaldehyde azine was prepared by the condensation reaction of 2-hydroxy-3methoxybenzaldehyde $(1.52 \mathrm{~g}, 1 \mathrm{mmol})$ and hydrazine hydrochloride $(0.34 \mathrm{~g}, 1 \mathrm{mmol})$ in ethanol $(30 \mathrm{~mL})$. The reaction mixture was refluxed and stirred at $70{ }^{\circ} \mathrm{C}$ for $24 \mathrm{~h}$. The resulting clear solution was kept in air and, after several minutes, yellow crystals were formed at the bottom of the vessel. The crystals were isolated, washed three times with ethanol and dried in an infrared-xay vacuum oven at $55^{\circ} \mathrm{C}$ (yield $72 \%$ ).

The IR spectra (4000-400 $\mathrm{cm}^{-1}$ ) were recorded as $\mathrm{KBr}$ pellets on a TG-209/Vector-22 spectrometer. ${ }^{1} \mathrm{H}$ NMR spectra was elucidated using a Bruker Avance AV $400 \mathrm{MHz}{ }^{1} \mathrm{H}$ NMR spectrometer. The solvent used was DMSO- $d_{6}$ and the chemical shifts were given in ppm in using TMS as the internal standard. The ${ }^{1} \mathrm{H}$ NMR spectra were taken at $25^{\circ} \mathrm{C}$. Mass spectra was obtained on a Agilent GC-MS 5975C instrument at $70 \mathrm{eV}$. XRD was obtained on a SMART APEXII Single-Crystal Diffractometer. The fluorescence spectra was obtained on a Varian LS55 fluorescence spectrometer. The solvent used was DMSO. Thermal analysis and differential thermogravimetric (TGA-DTG) analyses were carried out in temperature range from 25 to $600{ }^{\circ} \mathrm{C}$ in nitrogen atmosphere by TGA-Q50 thermal analyzer. The experimental conditions were: aluminium crucible with $1 \mathrm{mg}$ of sample, nitrogen atmosphere (nitrogen flow 
$50 \mathrm{~mL} / \mathrm{min}$ ) and heating rate of $20^{\circ} \mathrm{C} / \mathrm{min}$. Differential scanning calorimetric (DSC) analyses were carried out in temperature range from 25 to $500{ }^{\circ} \mathrm{C}$ in nitrogen atmosphere by DSC-Q20 analyzer. The experimental conditions were: aluminium crucible with $2 \mathrm{mg}$ of sample, nitrogen atmosphere (nitrogen flow $50 \mathrm{~mL} / \mathrm{min}$ ) and heating rate of $10{ }^{\circ} \mathrm{C} / \mathrm{min}$.

\section{RESULTS AND DISCUSSION}

The IR spectrum (Fig. 1) shows a broad band at $3470 \mathrm{~cm}^{-1}$, which may be assigned to the $-\mathrm{OH}$. The bands appeared at around $3000 \mathrm{~cm}^{-1}$ are assigned to the phenyl group and the strong intensity peak at $1615 \mathrm{~cm}^{-1}$ belongs to $v(C=N)$, suggesting that the condensation was carried out and producted the hydrazone. The peak at $1257 \mathrm{~cm}^{-1}$ is the usual mode of $\mathrm{C}-\mathrm{O}$ bending vibration. Bands appearing at 1577, 1460, 777, 731 $\mathrm{cm}^{-1}$ are the usual modes of phenyl ring vibrations.

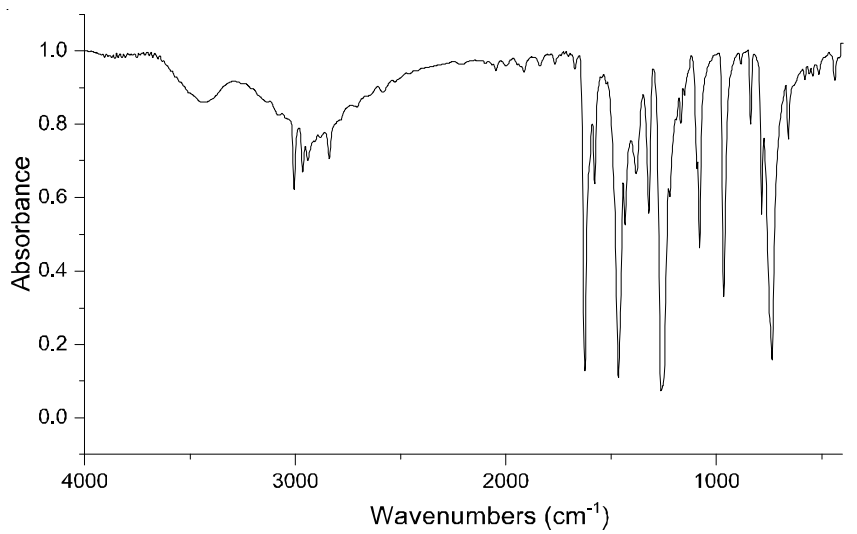

Fig. 1. Infrared spectrum of the (E,E)-2-hydroxy-3-methoxybenzaldehyde azine

The ${ }^{1} \mathrm{H}$ NMR spectrum of the hydrazone (Fig. 2) show a singlet band at $3.83 \mathrm{ppm}$ that was assigned to the proton of $-\mathrm{OCH}_{3}$ group. The multiplet signals at 6.87-7.29 ppm were attributed to the protons of aromatic rings. The signal of the azomethine group was observed at $8.97 \mathrm{ppm}$, which could further prove the formation of the hydrazone and the signal of the $-\mathrm{OH}$ was found at $10.85 \mathrm{ppm}$.

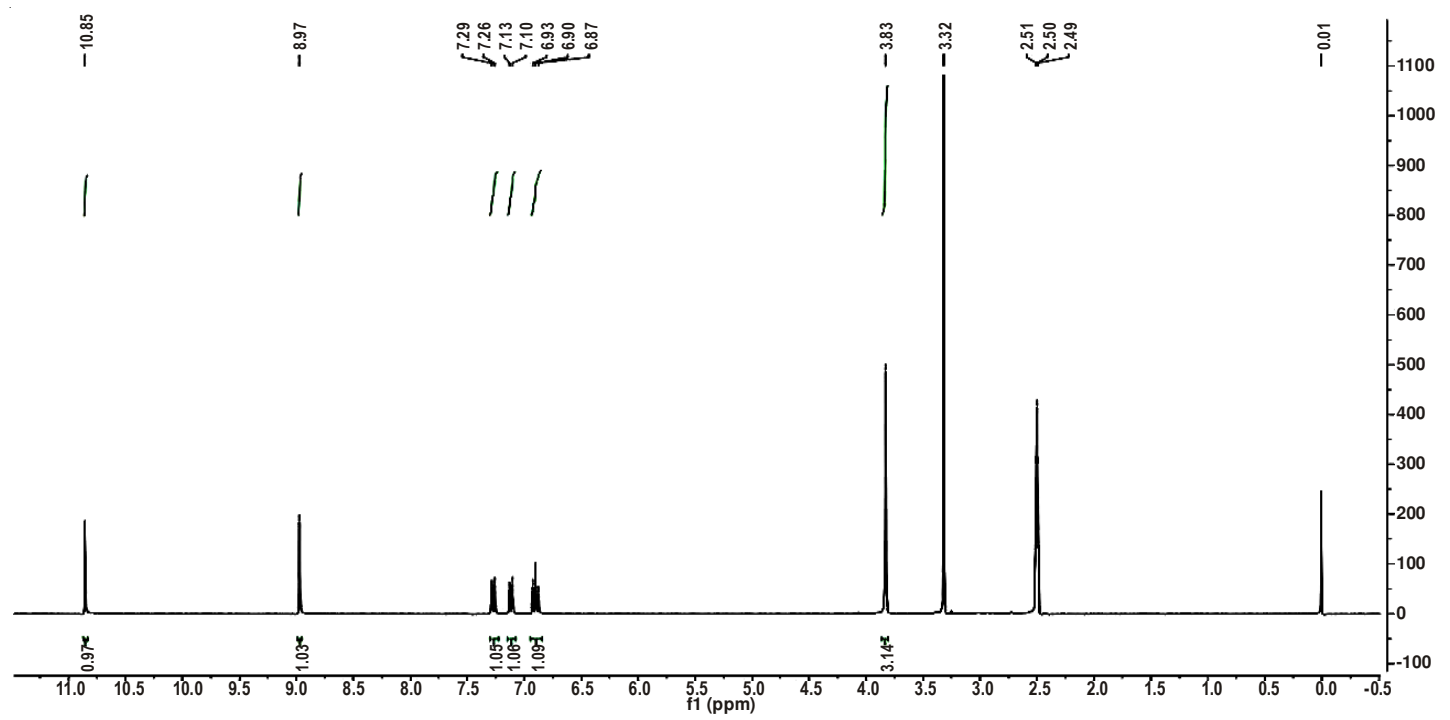

Fig. 2. ${ }^{1} \mathrm{H}$ NMR spectral of the (E,E)-2-hydroxy-3-methoxybenzaldehyde azine

Mass Spectroscopy: The most significant $\mathrm{m} / \mathrm{z}$ peaks in Fig. 3 of mass spectrum of the hydrazone are given in Table-1.

\section{TABLE-1}

MASS SPECTRUM $(m / z)$ OF THE $(E, E)-2-H Y D R O X Y$ -3-METHOXYBENZALDEHYDE AZINE

$\mathrm{C}_{16} \mathrm{H}_{16} \mathrm{~N}_{2} \mathrm{O}_{4}$

$\begin{array}{ll}\mathrm{C}_{16} \mathrm{H}_{16} \mathrm{~N}_{2} \mathrm{O}_{4} & 300 \\ {\left[\mathrm{C}_{16} \mathrm{H}_{15} \mathrm{~N}_{2} \mathrm{O}_{3}\right]^{+}} & 283\end{array}$

$\left[\mathrm{C}_{8} \mathrm{H}_{8} \mathrm{NO}_{2}\right]^{+} \quad 150$

$\left[\mathrm{C}_{7} \mathrm{H}_{5} \mathrm{NO}_{2}\right]^{+}$

$\left[\mathrm{C}_{6} \mathrm{H}_{4} \mathrm{O}_{2}\right]^{+} \quad 108$

$\left[\mathrm{C}_{5} \mathrm{H}_{4} \mathrm{O}\right]^{+}$

$\left[\mathrm{C}_{3} \mathrm{O}\right]^{+}$ 52

The (E,E)-2-hydroxy-3-methoxybenzaldehyde azine show one peak at $m / z, 300$ assigned to the $\mathrm{C}_{16} \mathrm{H}_{16} \mathrm{~N}_{2} \mathrm{O}_{4}$ molecular ion. It also shows a series of peaks i.e. $\mathrm{m} / \mathrm{z}(\%): 300$ (81.55), 283 (26.46), 150 (50.34), 135 (65.40), 80 (51.35) and 52 (100) corresponding to various fragments. Their signals give an idea about the formation of the hydrazone (Scheme-II).

$\mathrm{X}$-ray structure: The X-ray structure of the $(E, E)-2-$ hydroxy-3-methoxybenzaldehyde azine is shown in Fig. 4 and selected metric parameters are listed in Table-2. The whole molecule is to be almost planar. There are two half-molecules in the asymmetric unit, the mid-points of the N-N bonds lie on centers of symmetry. The molecular structure is stabilized by an intermolecular $\mathrm{O}-\mathrm{H} \cdots \mathrm{N}$ hydrogen bond which forms a six-membered ring.

Other X-ray crystallographic data are shown below: Monoclinic, space group P2(1)/n, $\mathrm{a}=5.9986(6), \mathrm{b}=18.3183$ (19), $c=6.8263(7) \AA ; \alpha=90, \beta=107.093(3), \gamma=90^{\circ} ; \mathrm{V}=$ 716.97(13) $\AA^{3} ; \mathrm{Z}=4$; $\mathrm{D}_{\text {calc. }}=1.391 \mathrm{mg} \mathrm{m}^{-3}, \mathrm{~F}(000)=316$; $\mu(\mathrm{Mo}-\mathrm{K} \alpha)=0.71073 ; \mathrm{T}=293$ (2) K.

Fluorescence spectroscopy: Fig. 5 shows the fluorescence spectra in the range $200-500 \mathrm{~nm}$ of the hydrazone in DMSO $\left(1 \times 10^{-4} \mathrm{~mol} \mathrm{~L}^{-1}\right)$. The emission spectrum indicates a peak at $275 \mathrm{~nm}$ due to $\pi-\pi^{*}$ transition, with the red shift deriving from the resonance donating effect of both $-\mathrm{OH}$ and $-\mathrm{OCH}_{3}$ groups in the phenyl ring and another broad peak at $425 \mathrm{~nm}$ is due to $n-\pi^{*}$ transition. 


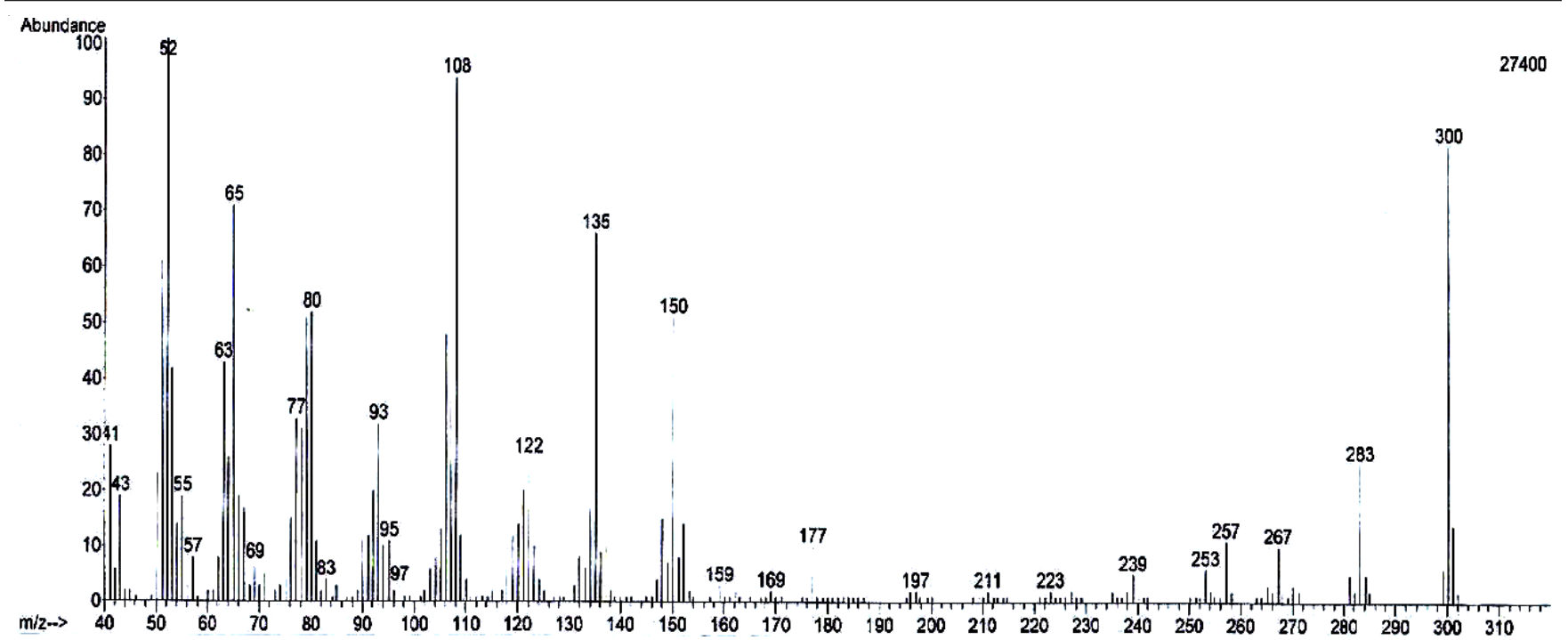

Fig. 3. Mass spectrum of the (E,E)-2-hydroxy-3-methoxybenzaldehyde azine

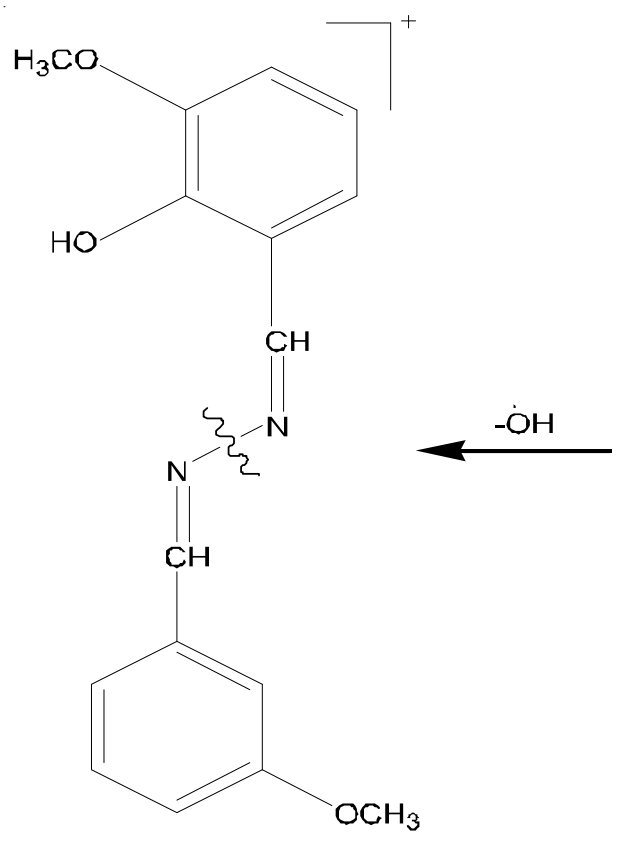

$\mathrm{m} / \mathrm{z} 283$

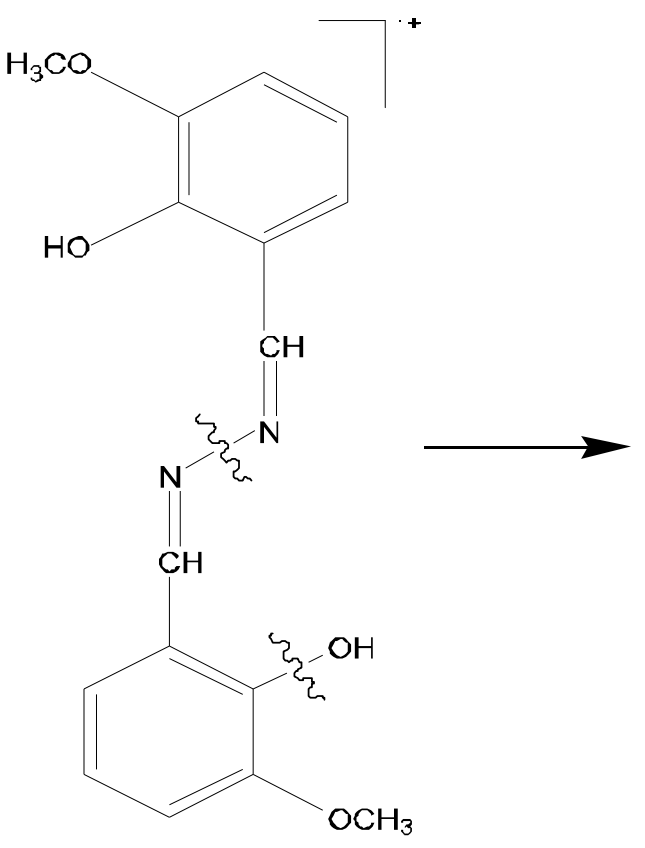

$\mathrm{m} / \mathrm{z} 300$

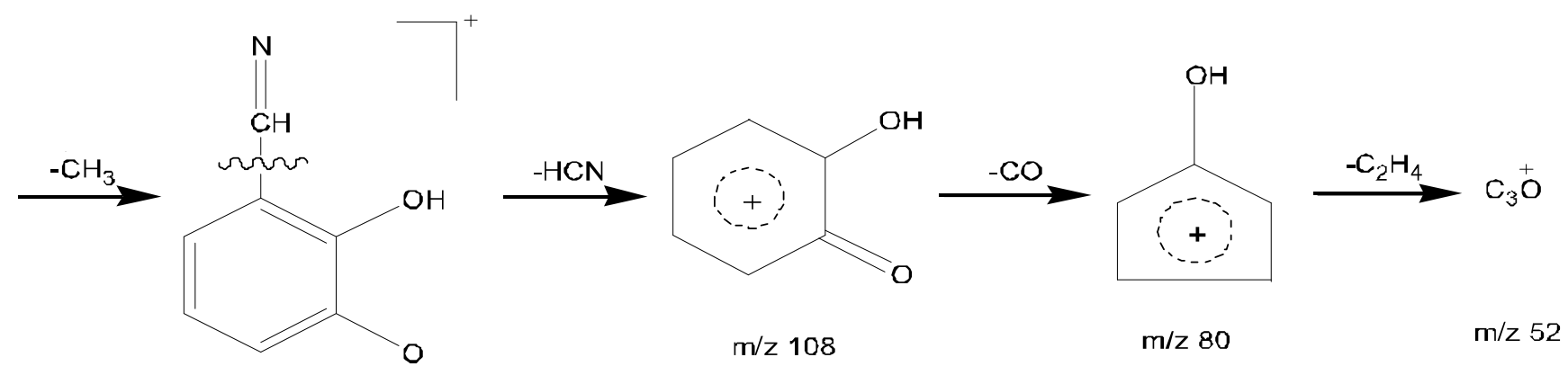

$\mathrm{m} / \mathrm{z} 135$

Scheme-II: Fragment patterns of the (E,E)-2-hydroxy-3-methoxy-benzaldehyde azine 


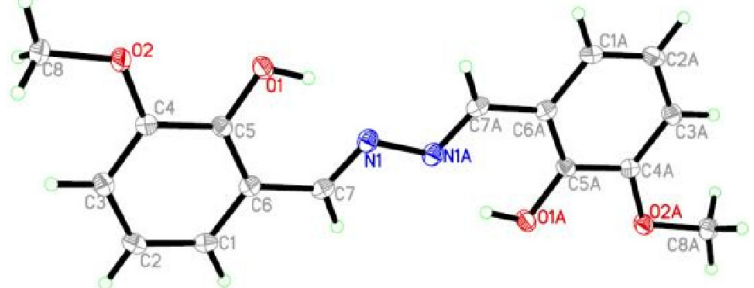

Fig. 4. Molecular structure of the (E, E)-2-hydroxy-3-methoxybenzaldehyde azine

\begin{tabular}{|c|c|c|c|}
\hline \multicolumn{4}{|c|}{$\begin{array}{c}\text { TABLE- } 2 \\
\text { SELECTED BOND LENGTHS AND BOND } \\
\text { ANGELS FOR THE COMPOUND }\end{array}$} \\
\hline Bond & Bond length $(\AA)$ & Bond & Bond angle $\left(^{\circ}\right)$ \\
\hline N1-N1A & $1.402(3)$ & C7-N1-N1A & $112.97(18)$ \\
\hline N1-C7 & $1.281(2)$ & N1-C7-C6 & $122.22(16)$ \\
\hline C7-C6 & $1.448(2)$ & C1-C6-C7 & $119.12(16)$ \\
\hline $\mathrm{C}(1)-\mathrm{C}(2)$ & $1.370(3)$ & C5-C6-C7 & $121.51(16)$ \\
\hline $\mathrm{C}(1)-\mathrm{C}(6)$ & $1.400(2)$ & C1-C6-C5 & $119.37(16)$ \\
\hline $\mathrm{C}(2)-\mathrm{C}(3)$ & $1.393(2)$ & C6-C5-C4 & $119.41(16)$ \\
\hline $\mathrm{C}(3)-\mathrm{C}(4)$ & $1.377(2)$ & $\mathrm{C} 5-\mathrm{C} 4-\mathrm{C} 3$ & $120.02(16)$ \\
\hline $\mathrm{C}(4)-\mathrm{C}(5)$ & $1.402(2)$ & $\mathrm{C} 4-\mathrm{C} 3-\mathrm{C} 2$ & $120.45(17)$ \\
\hline $\mathrm{C}(5)-\mathrm{C}(6)$ & $1.402(2)$ & $\mathrm{C} 3-\mathrm{C} 2-\mathrm{C} 1$ & $120.12(17)$ \\
\hline
\end{tabular}

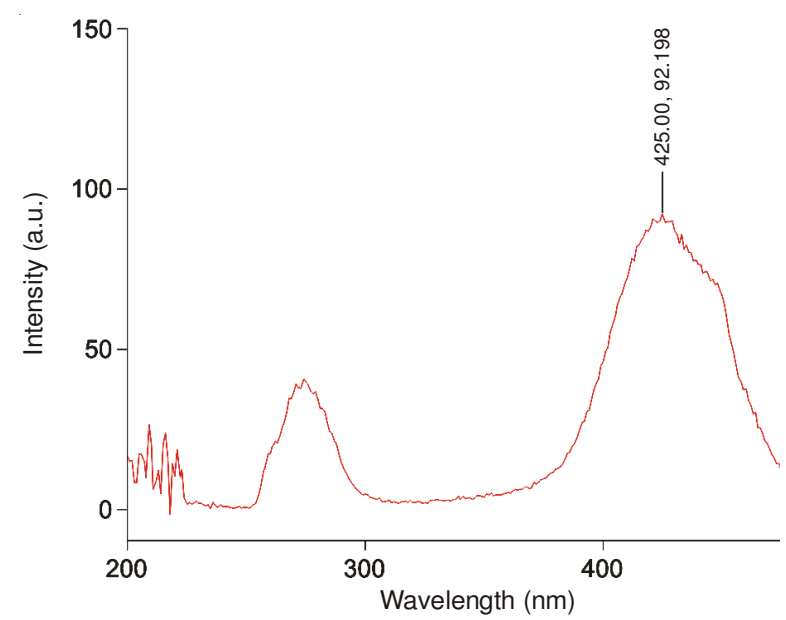

Fig. 5. Emission spectrum of the $(E, E)$-2-hydroxy-3-methoxybenzaldehyde azine

TG/DTA-DSC analysis: The TG thermogram of the hydrazone (Fig. 6) shows only one stage of decomposition from 198 to $315{ }^{\circ} \mathrm{C}$ with about weight loss $100 \%$ and the fastest rate point of the mass loss is at $308^{\circ} \mathrm{C}$ in the DTG (Fig. 6).

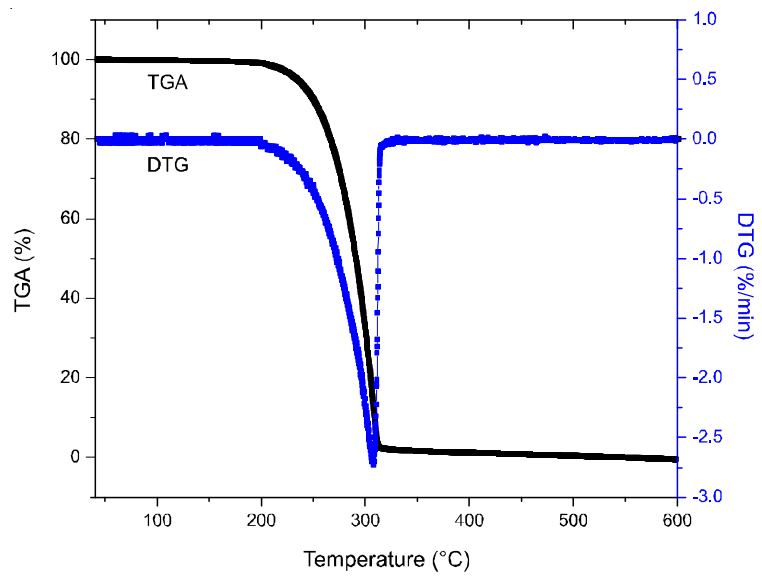

Fig. 6. TG-DTG of the (E,E)-2-hydroxy-3-methoxybenzaldehyde azine
The DSC curve (Fig. 7) indicates that the melt point of the hydrazone is $185-187^{\circ} \mathrm{C}$ and the maximum peak at $205^{\circ} \mathrm{C}$ in DSC is observed by endothermic reaction.

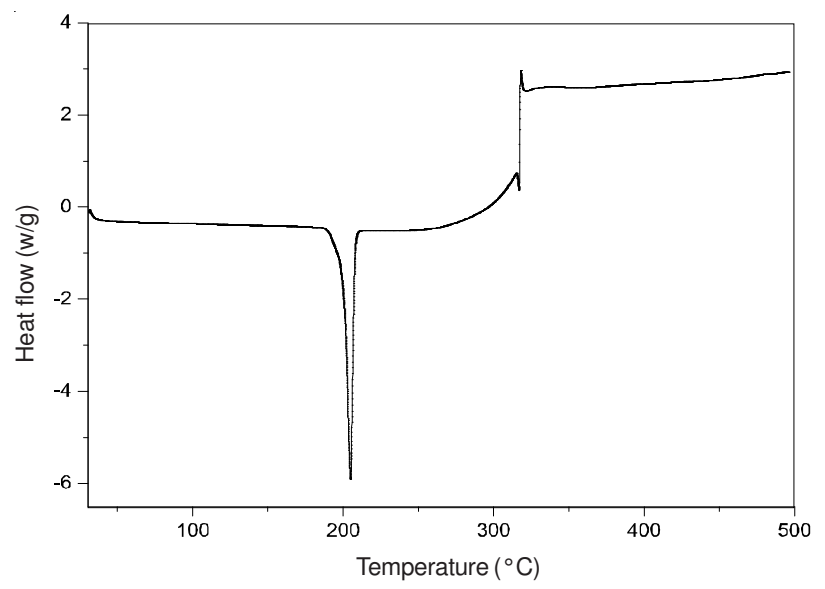

Fig. 7. DSC of the (E,E)-2-hydroxy-3-methoxybenzaldehyde azine

\section{Conclusion}

In summary, the (E,E)-2-hydroxy-3-methoxybenzaldehyde azine was synthesized by the condensation reaction in a relatively good yield. Its spectroscopic characterizations were analyzed in terms of IR, ${ }^{1} \mathrm{H}$ NMR, MS, XRD, fluorescence spectrometry and the thermal stability was also studied.

\section{ACKNOWLEDGEMENTS}

The authors gratefully acknowledged the National Natural Science Foundation of China (21261024), Jiangxi Department of Education (GJJ11704, GJJ12603), the Natural Science Foundation of Jiangxi Province (20132BAB203002) and Key Laboratory of Jiangxi Province for Research on Active Ingredients in Natural Medicines (China) for financial support.

\section{REFERENCES}

1. M.S. Refat and and A. A. Ibrahim, Spectrochim. Acta A, 70, 234 (2008).

2. D.F. Taber and P.F. Guo, J. Org. Chem., 73, 9479 (2008).

3. J. Chakraborty, R.K.B. Singh, B. Samanta, C.R. Choudhury, S.K. Dey, P. Talukder, M.J. Borah and S. Mitra, Z. Naturforsch., 61b, 1209 (2006).

4. A. Irshad, K. Misbah-ul-Ain and A. Makshoof, J. Pure Appl. Sci., 17, 67 (1998).

5. S.P. Douglas and P.N. Rao, Int. J. Eng. Sci. Technol., 2, 4655 (2010).

6. N.H. Al-Sha'alan, Molecules, 12, 1080 (2007).

7. S.K. Sridhar, M. Saravanan and A. Ramesh, Eur. J. Med. Chem., 36, 615 (2001).

8. V. Alptüzün, S. Parlar, H. Tasli and E. Erciyas, Molecules, 14, 5203 (2009).

9. C. Mirela, I. Emilia and G. Rodica, Rev. Roum. Chim., 10, 911 (2008).

10. S.N. Pandeya, A.S. Raja and J.P. Stables, J. Pharm. Pharmaceut. Sci., 3, 266 (2002)

11. C.M. Moldovan, O. Oniga, A. Pârvu, B. Tiperciuc, P. Verite, A. Pîrnau, O. Crisan, M. Bojita and R. Pop, Eur. J. Med. Chem., 4, 526 (2011).

12. S. Gemma, G. Kukreja, C. Fattorusso, M. Persico, M.P. Romano, M. Altarelli, L. Savini, G. Campiani, E. Fattorusso, N. Basilico, D. Taramelli, V. Yardley and S. Butini, Bioorg. Med. Chem, Lett., 16, 5384 (2006).

13. A. Nayyar, A. Malde, E. Coutinho and R. Jain, Bioorg. Med. Chem., 14, 7302 (2006).

14. M.K. Mao and J.E. Franz, Synthesis, 920 (1991).

15. M. Böger, D. Dürr, L. Gsell, R. GHall, F. Karrer, O. Kristiansen, P. Maienfisch, A. Pascual and A. Rindlisbacher, Pest Manage. Sci., 57, 191 (2001).

16. J.B. Raoof, R. Ojani and Z. Mohammadpour, Int. J. Electrochem. Sci., 5, 177 (2010) 